\title{
Real-time imaging of ${ }^{35} \mathrm{~S}$-sulfate uptake in a rape seed plant
}

\author{
By T. M. Nakanishi*, M. Yamawaki, H. Ishibashi and K. Tanoi \\ Laboratory of Radioisotope Plant Physiology, Department of Applied Biological Chemistry, Graduate School of Agricultural and Life Sciences, \\ The University of Tokyo, 1-1-1 Yayoi, Bunkyo-ku, Tokyo, 113-8657, Japan
}

(Received November 30, 2009; accepted in revised form February 18, 2010)

\section{Real-time imaging system $/{ }^{35} \mathrm{~S} /{ }^{32} \mathrm{P} /$ Rape seed plant}

Summary. We present real-time images of ${ }^{35} \mathrm{~S}$-sulfate uptake in a rapeseed plant visualized by the system we developed. In the leaves of rapeseed plants, ${ }^{35} \mathrm{~S}$ accumulated in higher amounts and more rapidly in the more developed leaves. This real-time imaging system can be used to visualize the movement of both ${ }^{35} \mathrm{~S}$ and ${ }^{32} \mathrm{P}$ in the same plant. In the pods of rapeseed, images of ${ }^{35} \mathrm{~S}$ show that ${ }^{35} \mathrm{~S}$ accumulated mostly in the terminal parts; on the other hand ${ }^{32} \mathrm{P}$, when applied as ${ }^{32} \mathrm{P}$-phosphoric acid, accumulated in the middle part of the pods.

\section{Introduction}

Rapeseed (Brassica napus L.) is widely grown for its edible oil. The chemical composition of the oil is reported to change during the process of seed ripening [1]. The circadian rythm of gas exchange and transpiration rates in rapeseed leaves and pods are related to the supply of nitrogen [2], and the amounts of nitrogenous fertilizer and the timing of application are important factors for increasing oil production [3]. The yield of oil differs from country to country even when the same cultivar is used [4], suggesting that discovering the optimum nutrient supply conditions for rapeseed growth is more important for increasing the amount of oil production than producing new plants through genetic engineering.

Plants need certain elements (nutrients) to grow and reproduce. However, hardly any studies have investigated how much of each nutritional element is absorbed at each stage of a plant's development. There are 17 elements essential for plant growth, and analyzing the amounts absorbed and the absorption process of each element will enable researchers to propose efficient and optimum nutrient conditions for obtaining high yields. The best way to analyze real-time plant activity of nutrient uptake is by applying a radioactive tracer, since this method allows nondestructive imaging as well as quantitative treatment of the images. When particular radioisotopes are supplied to a plant, it is possible to determine the elemental profile within the plant.

Recently, imaging techniques have dramatically developed to the point that they now allow imaging of chem-

*Author for correspondence

(E-mail: atomoko@mail.ecc.u-tokyo.ac.jp). icals or ions not only within a single cell but also within the organelles of a cell. Most of these imaging techniques employ fluorescent dyes or probes. However, measurements of fluorescence can be performed only under dark conditions and images cannot be detected in the light, which makes imaging of living plants problematic. In the case of radioisotopes, on the other hand, imaging of the radiation from the plant is possible even under light conditions, so natural plant activity can be maintained under an appropriate light/dark cycle. Another advantage of radioisotope imaging is that, because the image is based on the radioactivity count, the brightness of the image can be calibrated to the elemental amount in the sample, whereas in the case of fluorescent imaging, it is very difficult to calibrate the intensity of the fluorescence in the image to the amount of the fluorescenceemitting chemical in the sample. Because of the advantages of radioisotope imaging of plants, we developed a real-time radioisotope imaging system in which the elemental profile is shown in successive digital images [5-9]. In plants, sulfate $\left(\mathrm{SO}_{4}{ }^{2-}\right)$ plays an important role under conditions of stress. In this study, we applied our radioisotope imaging system to examine how ${ }^{35} \mathrm{~S}$-sulfate is taken up by a rapeseed plant.

\section{Materials and Methods}

The basic system we developed is illustrated in Fig. 1. A radiolabeled chemical is supplied to the plant, and the aboveground parts of the plant are irradiated with light. The beta-rays emitted from the sample are converted to

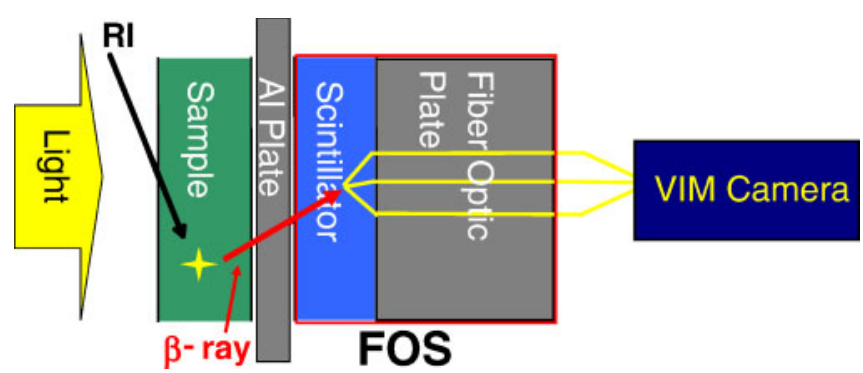

Fig. 1. Basic structure of real-time imaging system. Beta-rays emitted from the radioisotope (RI) in the sample impinge on the fiber-optic scintillator (FOS) where they are converted to light by a CsI(Tl) scintillator and directed via a fiber optic plate to a highly sensitive CCD camera (video-intensified microscopy [VIM] camera). 
light by a fiber-optic scintillator (FOS) comprising a CsI(Tl) scintillator combined with a fiber optic plate (microchannel plate, MCP). The weak light from the scintillator is guided through the fiber optic plate to a video-intensified microscopy (VIM) unit where it is amplified by an image intensifier (I.I. Unit; Hamamatsu Photonics, Co., Japan) and recorded with a highly sensitive CCD camera.

The seeds of rapeseed plants (B. napus L. cv. "Kizakinonatane" and "Kirariboshi") were washed well and germinated on wet paper under darkness. The seedlings were grown in a sulfate-deficient MGRL culture solution for $18 \mathrm{~d}$. The seedlings were then transferred to a vessel containing $10 \mathrm{~mL}$ of MGRL culture solution containing $1.5 \mathrm{mM}$ of ${ }^{35} \mathrm{~S}$ sulfuric acid $(3 \mathrm{MBq} / 10 \mathrm{~mL})$. The FOS was placed as close as possible to the aboveground parts of the plant, and the radiation image of the sample was recorded with the CCD camera in the dark. ${ }^{35} \mathrm{~S}$ images of the plant were were taken at an interval of $8 \mathrm{~min}$ for $960 \mathrm{~min}$.

To obtain images of the accumulation of sulfate within seedpods, we harvested whole "Kizakinonatane" plants from a field planted at Tanashi Experimental Farm, Tokyo, Japan, at a time when the seedpods were well developed. The roots of the plant were removed, and MGRL culture solution containing $1.5 \mathrm{mM}$ of sulfuric acid and its tracer ( $3 \mathrm{MBq} / 20 \mathrm{~mL}$ ) was supplied to the plant from the bottom part of the stem for $20 \mathrm{~h}$. Then, the plant was removed from the culture solution and placed on an imaging plate (IP) and exposed for $22 \mathrm{~h}$. The image on the IP was scanned by a scanner (Fra5000; Fuji Film, Co., Japan).

Whole "Kizakinonatane" plants at a similar growth stage were also harvested from the field to perform real-time imaging. The roots were removed and MGRL culture solution containing sulfuric acid and its tracer $(3 \mathrm{MBq} / 20 \mathrm{~mL})$ was supplied to the plant from the bottom part of the stem. Successive images of ${ }^{35} \mathrm{~S}$ were taken for $1500 \mathrm{~min}$ in the same way as cited above for seedlings.

To perform real-time imaging of both ${ }^{35} \mathrm{~S}$ and ${ }^{32} \mathrm{P}$ in the same plant, rapeseed plants (B. napus L. cv. "Westar") were grown in an MGRL culture solution for $82 \mathrm{~d}$ until the pods were developed. Then $0.15 \mathrm{mM}$ of sulfuric acid with its tracer $(10 \mathrm{MBq} / 5 \mathrm{~mL})$ was supplied to the plant from the roots, and uptake of ${ }^{35} \mathrm{~S}$ in pod parts was visualized by the real-time imaging system. The images were accumulated every $10 \mathrm{~min}$ for $4000 \mathrm{~min}$. After ${ }^{35} \mathrm{~S}$ images had been accumulated, detection of ${ }^{35} \mathrm{~S}$ in the pod was minimized by increasing the CCD camera's discrimination threshold for image acquisition. Above a discrimination threshold of 100 , an ${ }^{35} \mathrm{~S}$ image was not detected. Under these imaging conditions, MGRL solution containing ${ }^{32} \mathrm{P}$ phosphoric acid $(1.0 \mathrm{MBq} / 5 \mathrm{~mL})$ was then supplied to the same plant in order to visualize only the ${ }^{32} \mathrm{P}$ in a plant that contained both ${ }^{35} \mathrm{~S}$ and ${ }^{32} \mathrm{P}$. Real-time images of the uptake of ${ }^{32} \mathrm{P}$-phosphoric acid in pods were obtained for a further $4000 \mathrm{~min}$.

\section{Results}

Fig. 2 shows the manner of ${ }^{35} \mathrm{~S}$-sulfate uptake in 18-day-old plants. A faint image of ${ }^{35} \mathrm{~S}$ appeared after $240 \mathrm{~min}$ of appli-
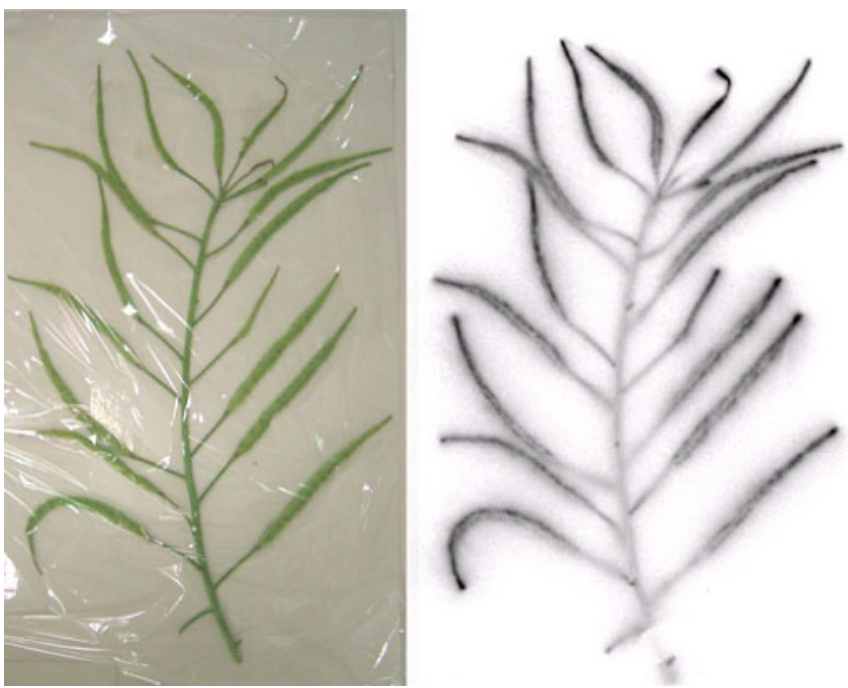

Fig. 3. ${ }^{35} \mathrm{~S}$-sulfuric acid distribution in pods visualized by imaging plate (IP). ${ }^{35} \mathrm{~S}$-sulfuric acid was supplied to Kizakinonatane and was exposed to an IP for $22 \mathrm{~h}$. Higher accumulation of ${ }^{35} \mathrm{~S}$ was shown at the terminal part of the pod.
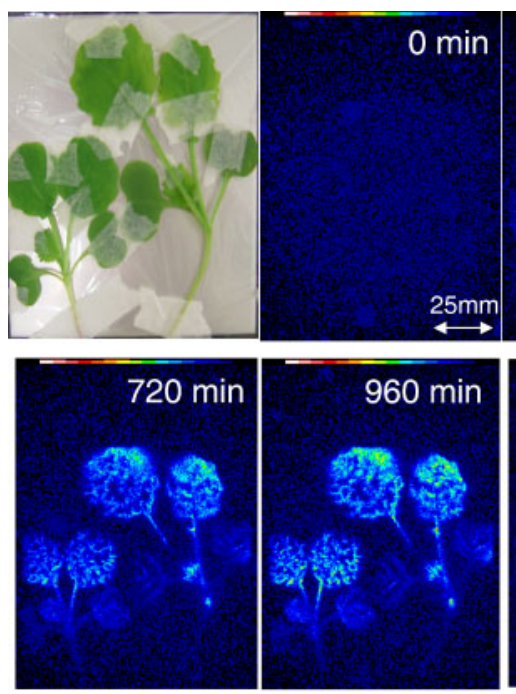

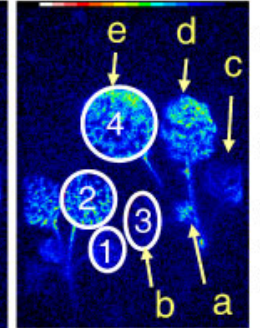

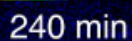
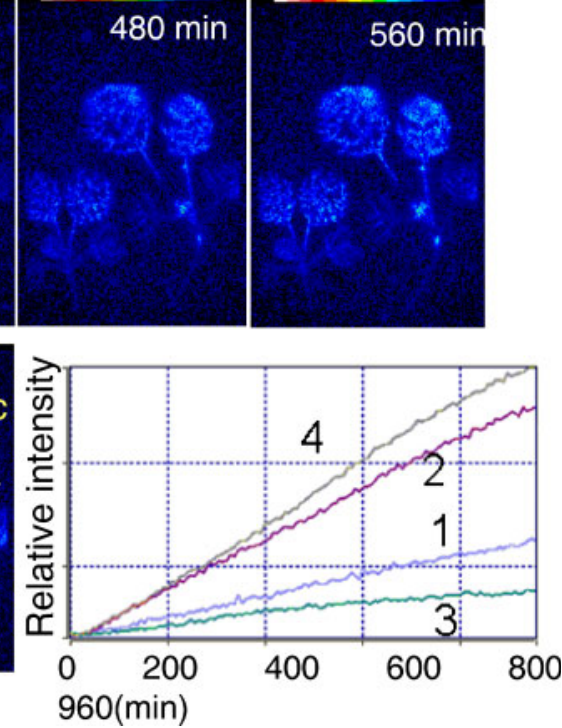

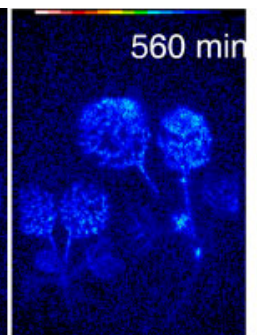

Fig. 2. ${ }^{35} \mathrm{~S}$-sulfuric acid uptake by rapeseed seedlings visualized by real-time imaging system. ${ }^{35} \mathrm{~S}$-sulfate uptake in 18-day-old plants. On the left is the cultivar "Kizakinonatane" and on the right is "Kirariboshi". The relative amounts of radioactivity in each leaf in "Kirariboshi" from youngest leaf to most developed leaf, as noted by labels "a" to "e", were $2.5,3.6,5.4,30$, and 42 , showing that ${ }^{35} \mathrm{~S}$ was accumulated to higher levels in the more developed leaves. The accumulation over time in leaves labeled 1 to 4 is plotted in the graph. 

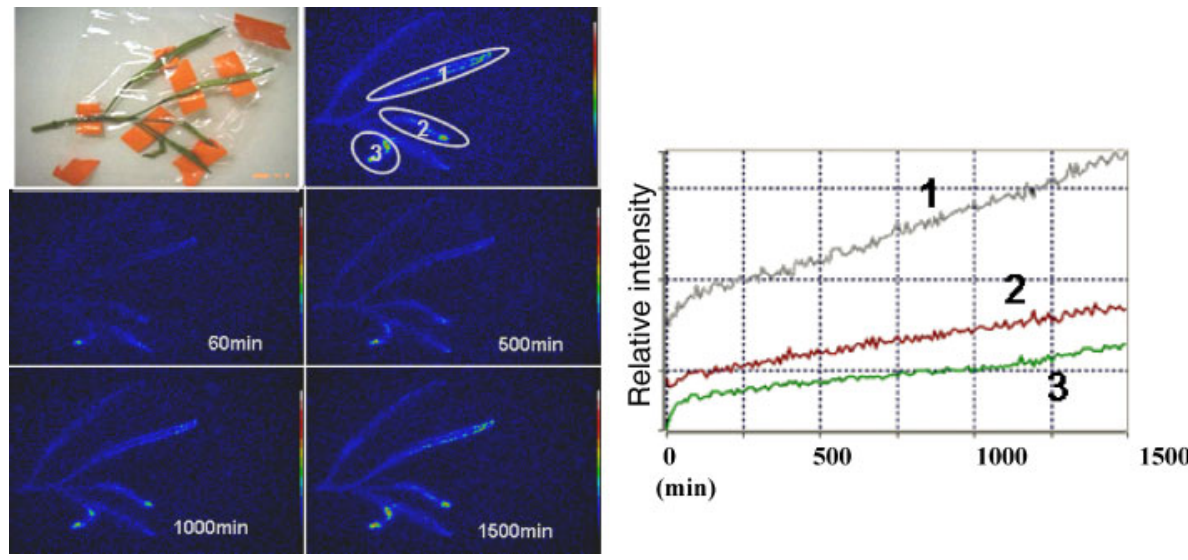

Fig. 4. ${ }^{35} \mathrm{~S}$-sulfuric acid uptake in pods visualized by real-time imaging system. To visualize accumulation manner of ${ }^{35} \mathrm{~S}$ in pods when ${ }^{35} \mathrm{~S}$-sulfuric acid was supplied to "Kizakinonatane", accumulation in pods was measured for $1500 \mathrm{~min}$. The accumulation over time in areas of the image labeled from 1 to 3 is plotted in the graph.

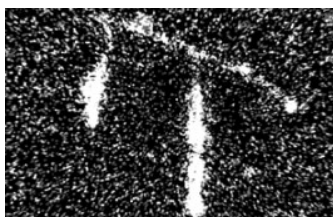

10

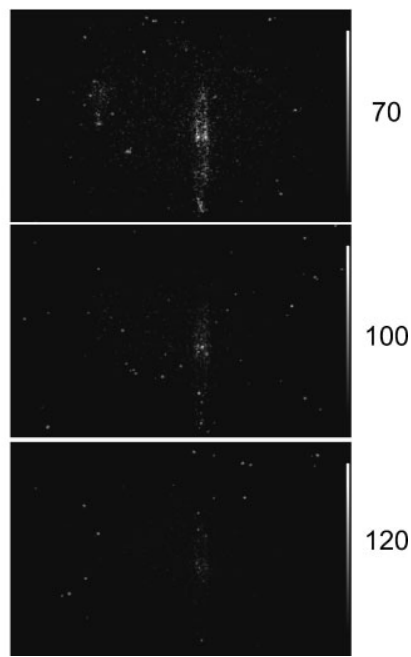

Fig. 5. Suppressing ${ }^{35} \mathrm{~S}$ image by increasing threshold value for detection of radioactivity from the sample. The threshold value was changed from 10 to 120 to minimize the ${ }^{35} \mathrm{~S}$ image. In order to suppress the ${ }^{35} \mathrm{~S}$ image and acquire only the ${ }^{32} \mathrm{P}$ image, a threshold value of 100 was employed.

cation, and the radioactivity in leaves increased with time. To analyze the manner of ${ }^{35} \mathrm{~S}$ uptake in the plants in more detail, we plotted the increases in radioactivity with time in the younger leaves of each plant (1 and 3) and the most expanded leaves (2 and 4) (Fig. 2). The uptake of ${ }^{35} \mathrm{~S}$ was faster in the more developed leaves, suggesting that the accumulation amount increased with the development of the leaves in each plant, and the increase with time was rather linear.

To visualize the pattern of ${ }^{35} \mathrm{~S}$ accumulation in pods, ${ }^{35} \mathrm{~S}$ sulfuric acid was supplied to "Kizakinonatane" and an IP was used to obtain the accumulation profile of ${ }^{35} \mathrm{~S}$. After $22 \mathrm{~h}$ of exposure to the IP, it was found that ${ }^{35} \mathrm{~S}$ tended to accumulate in higher amounts in the terminal parts of the pods (Fig. 3). To visualize the real-time accumulation of ${ }^{35} \mathrm{~S}$ in the pods in more detail, the root part of the plant was removed and ${ }^{35} \mathrm{~S}$-sulfuric acid solution was supplied to the plant from the bottom part of the stem. The images taken by the real-time imaging system show higher amounts of ${ }^{35} \mathrm{~S}$ in the terminal parts of the pods (Fig. 4), the same tendency as shown in the image taken by the IP (Fig. 3). For the images in Fig. 4, the discrimination level of the detector was set to 10. When this discrimination level was increased to 120 , all the ${ }^{35} \mathrm{~S}$ images disappeared (Fig. 5). To confirm this imag-

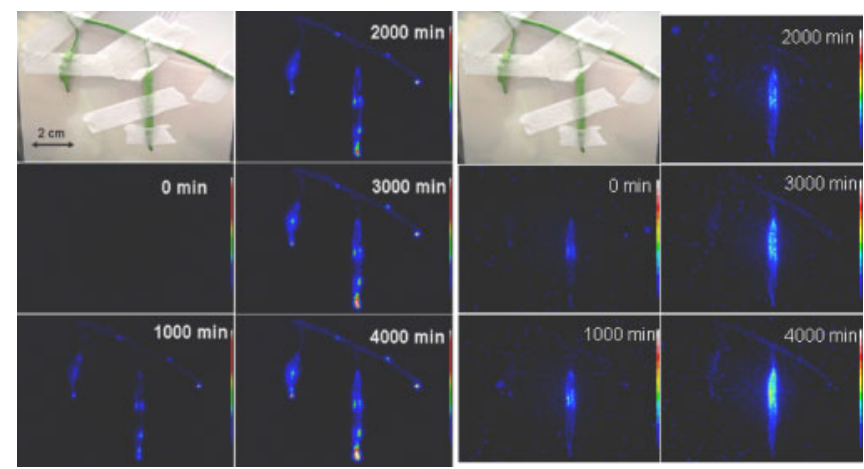

Fig. 6. ${ }^{35} \mathrm{~S}$-sulfuric acid and ${ }^{32} \mathrm{P}$-phosphoric acid uptake in pods by real-time imaging system. There was a difference in the manner of accumulation between ${ }^{35} \mathrm{~S}$ and ${ }^{32} \mathrm{P} .{ }^{32} \mathrm{P}$ was shown to accumulate in the middle part of the pods (set of images on the right), whereas ${ }^{35} \mathrm{~S}$ accumulated in the terminal part of the pods (left).

ing condition, we applied ${ }^{32} \mathrm{P}$-phosphoric acid to the same sample. Because the beta-ray energy of ${ }^{32} \mathrm{P}$ is much higher than that of ${ }^{35} \mathrm{~S}$, setting the discrimination level to 100 allows us to visualize only the ${ }^{32} \mathrm{P}$ in the image. Successive images of ${ }^{32} \mathrm{P}$-phosphoric acid uptake showed that ${ }^{32} \mathrm{P}$ accumulated mostly in the middle parts of the pod (Fig. 6).

\section{Discussion}

To the best of the knowledge this is the first report to show real-time ${ }^{35} \mathrm{~S}$ imaging in a plant. Supplying the plant with ${ }^{35} \mathrm{~S}$-sulfuric acid allowed us to observe the manner of ${ }^{35} \mathrm{~S}$ accumulation in leaves, especially along the leaf veins. ${ }^{35} \mathrm{~S}$ tended to accumulate at the rim of the leaf, suggesting that ${ }^{35} \mathrm{~S}$ is easily translocated along with the movement of the transpiration stream. The uptake amount of ${ }^{35} \mathrm{~S}$ was higher in developed leaves than in younger leaves, suggesting that there was no preference for translocation of ${ }^{35} \mathrm{~S}$ to younger leaves. However, in the case of ${ }^{32} \mathrm{P}$, it has been found that ${ }^{32} \mathrm{P}$-phosphate taken up by a soybean plant was preferentially moved to younger tissues first and then translocated to older tissues [5,6]. Based on the images obtained, the resolution of the real-time imaging system was estimated to be about $100 \mu \mathrm{m}$.

When the translocation of ${ }^{35} \mathrm{~S}$ in rapeseed pods was visualized, it was found that there was a tendency for ${ }^{35} \mathrm{~S}$ to accumulate in the terminal parts of the pods. This also suggested 
that ${ }^{35} \mathrm{~S}$ was moved easily via the transpiration stream, from the proximal part to the terminal part of the pod. This tendency was confirmed both by the IP and by the real-time imaging system. In the case of the real-time imaging system, the FOS equipped with the $\mathrm{CsI}(\mathrm{Tl})$ scintillator was placed as close as possible to the plant sample. Although the pods were rather thin, it is not known how much of the radioactivity in the pod was counted to visualize. Strictly speaking, the radioactivity count changes with the distance between the ${ }^{35} \mathrm{~S}$ in the tissue sample and the FOS. To accurately determine the amount of ${ }^{35} \mathrm{~S}$, it is necessary to prepare the sample as flat as possible, and images of standard solutions of ${ }^{35} \mathrm{~S}$ sulfuric acid should be taken at the same time as the image of the sample. The intensity of the image can then be calibrated against the standard solutions, and the amount of $\mathrm{S}$ distributed among the tissues can thus be calculated. In the case of IP imaging, ${ }^{35} \mathrm{~S}$ activity in the images of different pod tissues was extracted and counted with a liquid scintillator. We found that about $78 \%$ of the ${ }^{35} \mathrm{~S}$ distributed in a pod accumulated in the wall of the pod , $2-3 \%$ in seeds, and $10-$ $11 \%$ in the central partition of the silique within the pods (data not shown).

The real-time accumulation images showed that the accumulation of ${ }^{35} \mathrm{~S}$ was higher in the terminal rims of developed leaves and pods than in younger tissues. Since the movement of ${ }^{35} \mathrm{~S}$ found in this study was different from that of ${ }^{32} \mathrm{P}$, we tried to compare the movement of both nuclides using the same sample. First of all, ${ }^{35} \mathrm{~S}$-sulfuric acid was supplied to the plant and the real-time imaging was performed. Then, to the same sample, ${ }^{32} \mathrm{P}$-phosphate was supplied and the accumulation manner was visualized by suppressing the ${ }^{35} \mathrm{~S}$ image by increasing the detector's discrimination threshold. When the detection of ${ }^{35} \mathrm{~S}$ was sufficiently decreased, only the real-time image of ${ }^{32} \mathrm{P}$ remained. Although ${ }^{35} \mathrm{~S}$ accumulated at the upper part in pods, ${ }^{32} \mathrm{P}$ tended to accumulate in the middle of the pod. Since there is a large difference between the beta-ray energies ${ }^{35} \mathrm{~S}$ and ${ }^{32} \mathrm{P}(167 \mathrm{keV}$ and $1711 \mathrm{keV}$, respectively), it was also possible to separate the images by applying an $\mathrm{Al}$ sheet in front of the scintillator to cut only the beta-rays from ${ }^{35} \mathrm{~S}$. An aluminum sheet of about $100 \mu \mathrm{m}$ in thickness was estimated to cut the beta-rays sufficiently from ${ }^{35} \mathrm{~S}$ and produce only a ${ }^{32} \mathrm{P}$ image (data not shown).

One disadvantage of radioisotope imaging is that it is based only on the radioactivity count emitted from the nuclide and it is not known in what chemical form the ra- dioisotope is incorporated into the plant. For example, the ${ }^{32} \mathrm{P}$-phosphate in the rapeseed plant might be converted to other chemicals. In the case of ${ }^{32} \mathrm{P}$ in a soybean plant, by chemical analysis of the tissue, we found that within $30 \mathrm{~min}$ inorganic phosphate was the main chemical form translocated from the roots to aboveground parts (data not shown).

The real-time imaging system allows us to visualize nutrient translocation in living plants, which is not well understood. Since there are many advantages of applying radioisotopes for imaging, we are now developing a system for real-time imaging at a microscopic level by introducing radioactivity measurement devices to one of the oculars of a fluorescence microscope.

Acknowledgment. This research was supported by Genesis Research Institute, Inc, in Japan.

\section{References}

1. Vera, C. L., Downey, R. K., Woods, S. M., Raney, J. P., McGregor, D. I., Elliott, R. H., Johnson, E. N.: Yield and quality of canola seed as affected by stage of maturity at swathing. Canadian J. Plant Sci. 87(1), 13-26 (2007).

2. Muller, J., Diepenbrock, W.: Measurement and modeling of gas exchange of leaves and pods of oil seed rape. Agric. Forest Meteorol. 139(3-4), 307-322 (2006).

3. Rathke, G. W., Behrens, T., Diepenbrock, W.: Integrated nitrogen management strategies to improve seed yield, oil content and nitrogen efficiency of winter oilseed rape (Brassica napus L.): a review. Agric. Ecosys. Environ. 117(2-3), 80-108 (2006).

4. Berry, P. M., Spink, J. H.: A physiological analysis of oilseed rape yields: past and future. J. Agricult. Sci. 144, 381-392 (2006).

5. Rai, H., Kanno, S., Hayashi, Y., Ohya, T., Nihei, N., Nakanishi, M. T.: Development of a real-time autoradiography system to analyze the movement of the compounds labeled with $\beta$-ray emitting nuclide in a living plant. Radioisotopes 57, 287-294 (2008).

6. Kanno, S., Rai, H., Ohya, T., Hayashi, Y., Tanoi, K., Nakanishi, M. T.: Real-time imaging of radioisotope compounds in a living plant. J. Radioanal. Nucl. Chem. 272(3), 565-570 (2007).

7. Nihei, N., Masuda, S., Rai, H., Nakanishi, M. T.: Imaging analysis of direct alanine uptake by rice seedlings. Radioisotopes 57, 361366 (2008).

8. Nakanishi, M. T., Yamawaki, M., Kanno, S., Nihei, N., Masuda, S., Tanoi, K.: Real-time imaging of ion uptake from root to above-ground part of the plant using conventional beta-ray emitters. J. Radioanal. Nucl. Chem. 282, 265-269 (2009).

9. Yamawaki, M., Kanno, S., Ishibashi, H., Noda, A., Hirose, A., Tanoi, K., Nakanishi, M. T.: The development of real-time RI imaging system for plant under light environment. J. Radioanal. Nucl. Chem. 282, 265-269 (2009). 